\title{
A new dedicated $\beta$-NMR/ $\beta$-NQR setup for LISE-GANIL
}

\author{
M. De Rydt ${ }^{a, *}$, R. Lozeva ${ }^{a, 1}$, N. Vermeulen ${ }^{a}$, \\ F. de Oliveira Santos ${ }^{b}$, S. Grévy ${ }^{b}$, P. Himpe ${ }^{a}$, C. Stödel ${ }^{b}$, \\ J.C. Thomas ${ }^{\mathrm{b}}$, P. Vingerhoets ${ }^{\mathrm{a}}$ and G. Neyens ${ }^{\mathrm{a}}$ \\ anstituut voor Kern- en Stralingsfysica, K.U. Leuven, Celestijnenlaan 200D, \\ B-3001 Leuven, Belgium \\ ${ }^{\mathrm{b}}$ Grand Accélérateur National d'Ions Lourds (GANIL), CEA/DSM-CNRS/IN2P3, \\ B.P. 55027, F-14076 Caen Cedex 5, France
}

\begin{abstract}
A new $\beta$-NMR/ $\beta$-NQR setup is developed to study nuclear magnetic dipole moments and electric quadrupole moments at the LISE fragment separator at GANIL. Two key elements make the new design very powerful: the homogeneous magnet and the strong radio-frequency amplifier. The combination of both makes it possible to examine a broad spectrum of magnetic dipole and electric quadrupole moments. Several factors have an influence on the detection efficiency and on the sensitivity of the setup to observe a resonant change in the $\beta$-asymmetry: the thickness and the tilting angle of the implantation crystal, the material in the vacuum chamber, the applied magnetic field and the $Q_{\beta}$-value of the decaying nuclei. A detailed study of these effects is made using a dedicated GEANT4 Monte Carlo simulation code.
\end{abstract}

Key words: nuclear moments, $\beta$-Nuclear Magnetic Resonance ( $\beta$-NMR), $\beta$-Nuclear Quadrupole Resonance ( $\beta$-NQR), detection system, GEANT4 PACS: 21.10.Ky, 27.20.+n, 25.70.Mn, 07.50.-e, 07.55.-w, 29.40.Mc, 07.20.Mc, 02.70.Uu

\footnotetext{
* Corresponding author

Email address: marieke.derydt@fys.kuleuven.be (M. De Rydt).

1 Present address: CSNSM, Université Paris-Sud 11, CNRS/IN2P3, F-91405 OrsayCampus, France
} 


\section{Introduction}

Many theoretical and experimental projects worldwide study the structure of exotic nuclei close to the drip lines. These nuclei are predicted to reveal new aspects of nuclear structure that challenge the existing models. The island of inversion $(N \simeq 20)$ and the neutron rich $N \simeq 28$ isotopes, for instance, exhibit unexpected changes from the shell-model configuration. Although only two examples are given here, structural evolution occurs around all 'traditional' magic numbers. For a recent review, see Ref. [1].

Magnetic dipole moments and electric quadrupole moments provide direct information on the nuclear structure. Therefore, both observables are highly suitable to study nuclei far from stability, where changes in the shell structure appear. The magnetic dipole moment, $\mu$, is very sensitive to the orbits occupied by the valence nucleons and it can reveal the details of the investigated nuclear configuration. The electric quadrupole moment, $Q$, is a direct measure of the nuclear deformation since it indicates the deviation of the nuclear charge distribution from a sphere.

In the past decades, remarkable progress has been made in producing highintensity beams of exotic nuclei at various accelerator facilities. Very neutronand proton-rich isotopes became available for nuclear structure research in general and for nuclear moment measurements in particular.

One of the most powerful methods to study ground-state magnetic dipole moments of radioactive isotopes is the $\beta$-Nuclear Magnetic Resonance $(\beta$ NMR) technique. Based on the same principle, the $\beta$-Nuclear Quadrupole Resonance $(\beta$-NQR) method was developed to study electric quadrupole moments. In literature, the term NQR usually refers to a technique which studies the quadrupole moment of stable nuclei based on the quadrupole interaction with their environment. For radioactive isotopes however, $Q$ is often measured through its interaction with an electric field gradient and an externally applied magnetic field. This method is called $\beta$-NQR, similar to the $\beta$-NMR technique which is used to study magnetic moments.

$\beta$-NMR as well as $\beta$-NQR require spin-polarized beams since they rely on a resonant change in the $\beta$-decay asymmetry. When the isotopes of interest are produced at an ISOL facility, the nuclear polarization can be obtained with optical laser pumping [2]. As this process strongly depends on the atomic properties of the nuclei involved, spin polarization can only be obtained for a limited amount of elements. In a projectile-fragmentation reaction, which is the production mechanism used at the LISE fragment separator at GANIL, the polarization is obtained by manipulating the reaction process. The angle put on the primary beam with respect to the entrance of the separator (typically $\left.2(1)^{\circ}\right)$ and the selection made in the longitudinal momentum distribution of the fragments result in a spin-polarized secondary beam [3-7].

As important and decisive information can be obtained from the measurement of nuclear moments, not only an accurate experimental technique but also a 
reliable setup is needed to study them. In the first section of this article, the $\beta$-NMR and $\beta$-NQR methods are discussed. In part two, a detailed description of a new $\beta$-NMR/ $\beta$-NQR setup, used at the LISE beam line, is given. The last section presents the GEANT4 simulation code and its applications, developed to evaluate the new setup.

\section{The $\beta$-NMR and $\beta$-NQR methods}

The $\beta$-NMR technique is used to measure the nuclear g-factor $g$, which is directly linked to the magnetic dipole moment via $\mu=g I \mu_{N}$, with $I$ the nuclear spin. In a $\beta$-NQR measurement, the quadrupole coupling constant $\nu_{Q}=\frac{e Q_{s} V_{z z}}{h}$ is determined, from which the spectroscopic quadrupole moment $Q_{s}$ can be deduced, provided that the electric field gradient $V_{z z}$ in the implantation crystal is known. Both techniques have been frequently used in the past and several different modes exist (e.g. the adiabatic fast passage method [8] or the continuous rf technique [9]). This work concentrates on the resonance methods that combine a constant implantation of polarized nuclei with a continuously applied radiofrequent (rf) field and a time-integrated detection of the ensemble polarization.

The $\beta$-NMR and $\beta$-NQR experiments are performed at the LISE fragment separator $[10,11]$. The isotopes of interest are produced in a projectile-fragmentation reaction, induced by a stable and fully stripped primary beam $(60-80 \mathrm{MeV} / \mathrm{u}$, 1-4 $\mathrm{e} \mu \mathrm{A}$ ) on a rotating target. The secondary beam is selected by two dipole stages and transported to the D6 focal point. The beam identification is performed with three Si-detectors along the beam path according to the standard energy loss versus time-of-flight method. A purification is done with the ${ }^{9} \mathrm{Be}$ wedge-degrader in the intermediate dispersive plane, the horizontal and vertical slits along the beam line and the Wien filter, installed in front of the $\beta$-NMR/ $\beta$-NQR setup. When all components are optimized, a secondary beam purity of more than $90 \%$ can be achieved. In order to maintain the reaction-induced polarization, the fragments need to be fully stripped and the electron pick-up along the beam path has to be reduced to a minimum by adapting the thickness of the target, the wedge and the degraders. According to the simulation code GLOBAL, embedded in LISE ++ package $[12,13]$, only fragments with $A<80$ are completely stripped after intermediate-energy projectile-fragmentation reactions.

At the end of the LISE fragment separator, the $\beta$-NMR/ $\beta$-NQR setup is installed. The polarized nuclei are implanted in a crystal after being energydegraded in two Al-foils. At $0^{\circ}$ and $180^{\circ}$ along the vertical polarization axis, above and below the implantation crystal, two pairs of plastic scintillators are positioned to detect the $\beta$-particles emitted when the implanted fragments decay (see Fig. 1). A spin-polarized ensemble of nuclei leads to an anisotropic 
$\beta$-decay pattern, given by the angular distribution function $W(\theta)[14]$ :

$$
W(\theta)=1+\frac{v_{\beta}}{c} A_{\beta} P \cos (\theta)
$$

$A_{\beta}$ is the asymmetry parameter that depends on the spin and the decay properties of the investigated nuclei. $P$ is the initial polarization of the implanted ensemble, induced by the nuclear reaction. The fraction $\frac{v_{\beta}}{c}$, with $v_{\beta}$ the velocity of the $\beta$-particles and $c$ the speed of light, is taken as 1 . The measured $\beta$-asymmetry $A$, which is related to the polarization in the ensemble, is then defined as

$$
A=\frac{N_{u p}-N_{\text {down }}}{N_{\text {up }}+N_{\text {down }}} \simeq A_{\beta} P
$$

where $N_{u p}$ is the number of coincident counts in the upper two detectors while $N_{\text {down }}$ denotes the number of coincidences in the lower set of scintillators. Going from a polarized to an unpolarized ensemble induces a $\beta$-asymmetry change proportional to $A_{\beta} P$. This change in asymmetry is observed in a $\beta$ NMR or $\beta$-NQR measurement since both methods use hyperfine interactions to resonantly destroy the polarization at a well-defined rf-frequency.

In both techniques, the stopper crystal is situated in the center of a static magnetic field $B_{0}$, which is also the center of the setup. The external magnetic field induces a Zeeman splitting of the nuclear $m$-states, such that the energy difference between two subsequent levels is proportional to the g-factor and the applied magnetic field.

$$
E_{m}-E_{m+1}=g \mu_{N} B_{0}=h \nu_{L}
$$

$\nu_{L}$ denotes the Larmor frequency and is typically of the order of 0.1 to $3 \mathrm{MHz}$. In $\beta$-NMR, only a Zeemann splitting of the nuclear levels is induced since an implantation crystal with a cubic lattice structure is chosen. In $\beta$-NQR, the polarized nuclei are implanted in a stopper with a non-cubic crystal structure. In that case, the quadrupole interaction with the electric field gradient causes an additional shift of the $m$-states which results in a non-equidistant level spacing. The energy difference between the magnetic sublevels $m$ and $m+1$ under the influence of a Zeemann and an axial symmetric quadrupole interaction can be calculated using perturbation theory. Up to the second order, the series expansion is given by expression (4), provided that the magnetic interaction (scaling with $\nu_{L}$ ) is much stronger than the quadrupole interaction (scaling with $\left.\frac{\nu_{Q}}{4 I(2 I-1)}\right)[8]$.

$$
\begin{gathered}
E_{m}-E_{m+1}=h \nu_{L}-\frac{3 h \nu_{Q}}{8 I(2 I-1)}(2 m+1)\left(3 \cos ^{2} \theta-1\right) \\
-\frac{h \nu_{Q}^{2}}{32 \nu_{L}} \frac{9}{4 I^{2}(2 I-1)^{2}}[6 m(m+1)-2 I(I+1)+3] \sin ^{4} \theta
\end{gathered}
$$

$\theta$ is the angle between the symmetry axis of the electric-field gradient in the crystal and the magnetic field $B_{0}$. 
Perpendicular to the static magnetic field, a radio-frequent magnetic field is applied inside a coil mounted around the implantation crystal (see Fig. 1, 2 and 3 ). During a $\beta$-NMR measurement, a particular frequency range is scanned in a number of discrete steps. For each step, the rf-frequency is continuously modulated around a central value to ensure that the resonance frequency is not missed. If the applied frequency range covers $\nu_{L}$ and if the rf-field strength is high enough, the occupation of all nuclear m-states is equalized and the polarization is destroyed. When the full rf-range has been scanned (typically in a few minutes), a measurement without rf-field is performed as a reference. This process is repeated until sufficient statistics are collected.

Multiple-rf $\beta$-NQR is based on the same principle, the only difference is the number of simultaneously applied frequencies. Expression 4 shows that, for a constant $\nu_{Q}$, subsequent $m$-states have different transition frequencies. For each value of $\nu_{Q}$, these correlated transition frequencies are applied simultaneously and each of them is modulated. When the quadrupole coupling constant is covered, the polarization is destroyed and a resonance is seen when the $\beta$ asymmetry is plotted as a function of the applied rf-frequency (see e.g. [15]).

\section{Details of the set-up}

When the secondary beam enters the $\beta$-NMR/ $\beta$-NQR setup, it first passes through two Al-degraders, placed at about $25 \mathrm{~cm}$ upstream from the implantation crystal. Behind the degraders, the fragments encounter a collimator and a Si-detector before entering the rf and detection region.

The variable degraders are two Al-foils with different thicknesses which can be inserted in the beam line and tilted with respect to the beam axis, allowing a total variable degrader thickness between $50 \mu \mathrm{m}$ and $2600 \mu \mathrm{m}$. Due to the variable thickness, the implantation energy of the fragments can be precisely tuned. In case the maximum thickness of $2600 \mu \mathrm{m}$ is not sufficient to stop the beam in the middle of the crystal (e.g. for very light nuclei), an extra Al-degrader can be mounted at a distance of a few $\mathrm{cm}$ from the crystal. By using an extra degrader instead of a thicker variable degrader, the angular divergence at large distances from the center is minimized.

Behind the set of variable degraders, a Pb-collimator is placed with a conical opening of $16 \mathrm{~mm}$. This collimator prevents scattered particles from the degraders to get into the detection region and it limits the diameter of the beam spot to $16 \mathrm{~mm}$.

Behind the collimator, the profile of the secondary beam can be measured using a position sensitive PIPS detector (Canberra, model PF20*20-500EB). This Si-detector is used during the beam tuning to check the transmission to the $\beta$-NMR/ $\beta$-NQR setup and to inspect the beam spot size and position in order to realize a correct implantation in the crystal. 
Behind the Si-detector, the secondary beam enters the rf and detection region. A schematic overview of this section is given in Fig. 1. The different parts, which are the magnet, the crystal holders, the rf-circuit and the $\beta$-detectors, will be further discussed in detail.

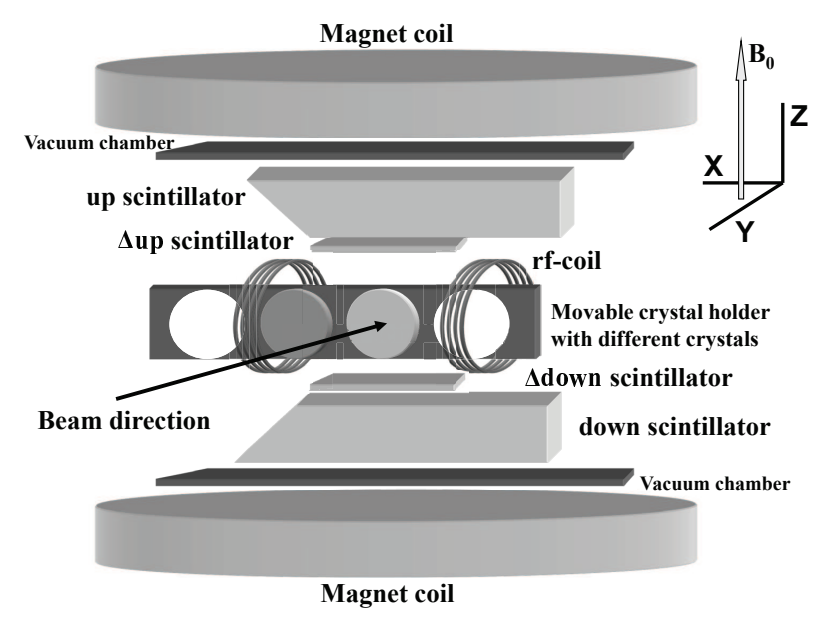

Fig. 1. A schematic overview of the rf and detection region of the newly designed setup.

\subsection{The magnet}

The static magnetic field $B_{0}$ is generated by an electromagnet of the type BE25v, manufactured by Bruker. The magnet has a variable pole gap, making it possible to achieve a central field of $2.2 \mathrm{~T}$ for an opening of $1 \mathrm{~cm}$ between the magnet poles. When the pole gap is expanded to its largest value $(14.6 \mathrm{~cm})$, the maximum field in the center drops to $0.87 \mathrm{~T}$. The $\beta$-NMR/ $\beta$-NQR application requires a $12 \mathrm{~cm}$ large gap since an aluminium vacuum chamber which contains the rf and detection region is inserted between the magnet poles. For this configuration, a maximum field of about $1.02 \mathrm{~T}$ can be obtained in the center.

Not only the range of $B_{0}$ but also the homogeneity of the magnetic field over the crystal dimensions is an important aspect in $\beta$-NMR/ $\beta$-NQR measurements. If the fragments experience very different field values over the implantation volume, a broadened resonance with reduced amplitude will be observed. Since the sensitivity of the method is proportional to the asymmetry change squared, a smaller amplitude due to the inhomogeneity of the magnetic field results in a less efficient technique and a significantly longer measuring time. Extensive field profile measurements in the three spatial directions have been carried out for magnetic fields of $0.18,0.5$ and $1 \mathrm{~T}$. A concise summary of these measurements is given in Table 1 which specifies the maximum deviation $\Delta B$ of the fields at a distance of $\pm 1 \mathrm{~cm}$ in the $\mathrm{X}, \mathrm{Y}$ and $\mathrm{Z}$ directions from the 
average value in the center. $\mathrm{X}, \mathrm{Y}$ and $\mathrm{Z}$ are defined in Fig. 1 and the center of the magnet is taken as the origin. A gradual change of the magnetic field has been observed in the $\mathrm{X}$ and $\mathrm{Y}$ directions. Going from the center to $\pm 1 \mathrm{~cm}$, the field decreases by only 0.02 to $0.03 \%$. In the $\mathrm{Z}$ direction, fluctuations occur, resulting in $\Delta B_{z} \leq 0.2 \%$. These fluctuations do not reduce the accuracy of the $\beta$-NMR/ $\beta$-NQR measurements since, according to the gaussian implantation profile of the fragments, most fragments are implanted close to the center $(<5 \mathrm{~mm})$ where $\Delta B_{z}$ is at most $0.05-0.09 \%$.

Table 1

Maximum deviation $\Delta \mathrm{B}(\%)$ of the magnetic field measured at $\pm 1 \mathrm{~cm}$ from the value in the center for three different magnetic fields.

\begin{tabular}{|c|c|c|c|}
\hline Direction & $\Delta B$ for $0.18 \mathrm{~T}$ & $\Delta B$ for $0.5 \mathrm{~T}$ & $\Delta B$ for $1 \mathrm{~T}$ \\
\hline $\mathrm{X}(\mathrm{Y}=\mathrm{Z}=0)$ & $0.02 \%$ & $0.02 \%$ & $0.03 \%$ \\
\hline $\mathrm{Y}(\mathrm{X}=\mathrm{Z}=0)$ & $0.02 \%$ & $0.03 \%$ & $0.03 \%$ \\
\hline $\mathrm{Z}(\mathrm{X}=\mathrm{Y}=0)$ & $0.17 \%$ & $0.11 \%$ & $0.09 \%$ \\
\hline
\end{tabular}

During the $\beta$-NMR or $\beta$-NQR run, the field is remotely set by a Labview application. After each frequency scan (which takes 5 to 10 minutes), a measurement of the magnetic field is performed by a hall probe (HP, Group3-Danfysik model MPT-141-25s communicating with a digital tesla meter DTM141) positioned at about $7 \mathrm{~cm}$ behind the crystal. The accuracy of the device is $2 \times 10^{-5} \mathrm{~T}$ for $B_{0}<1.2 \mathrm{~T}$. The measured magnetic field is compared to a reference value and adjusted when necessary using a feedback loop. Since the hall probe is positioned behind the crystal, an online measurement of the magnetic field in the center is not possible. However, the central value can be calculated using the field calibrations which are done before and after each experiment. During the calibration process, a complete and stable hysteresis curve is measured by varying the magnet current in steps of $0.5 \mathrm{~A}$. This procedure is performed in the center as well as at the position where the hall probe is placed during the experiment, firmly establishing the relation between both.

\subsection{The crystal holders}

For the $\beta$-NMR/ $\beta$-NQR experiments, two different crystal holders can be used. One operates at room temperature, the other is used to cool or heat the stopper material. On the room temperature crystal holder (Fig. 2), four different crystals can be mounted. The metal rod to which they are attached can be moved in and out the vacuum chamber, allowing another crystal to be placed in the beam path without breaking the vacuum. The holder can also be tilted with respect to the beam axis in order to minimize the path length of the $\beta$-particles inside the crystal before they reach the detectors. 


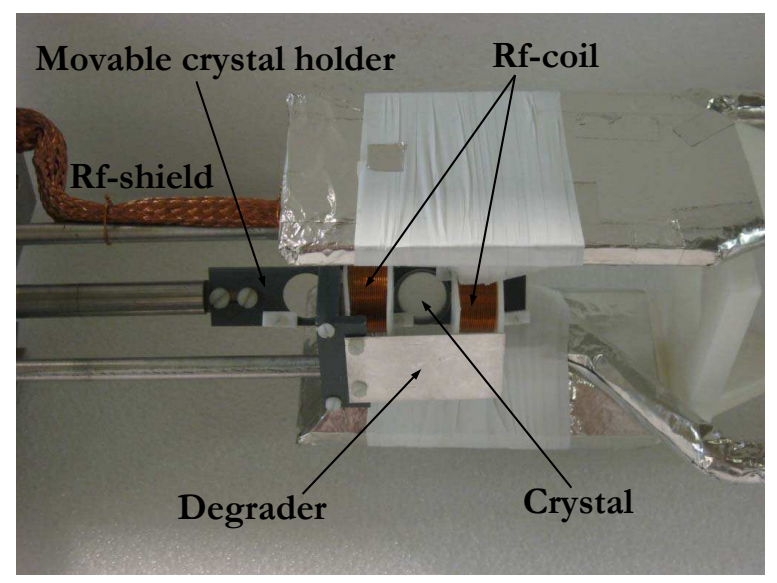

Fig. 2. Downstream view of the room temperature crystal holder.

If the half life of the implanted nuclei is longer than the relaxation time, the nuclear polarization is destroyed by random spin-lattice interactions in the crystal before the $\beta$-decay takes place [8]. This effect is often caused by fluctuations in the electric field gradient due to lattice vibrations and diffusing defects or by the energy transfer to electrons. As the spin-lattice $T_{1}$-relaxation time strongly depends on the temperature [16], the problem can be solved by cooling the implantation crystal (e.g. [17] for $\mathrm{NaCl}$ ). When cooling is applied, the cold finger crystal holder (Fig. 3) is used.

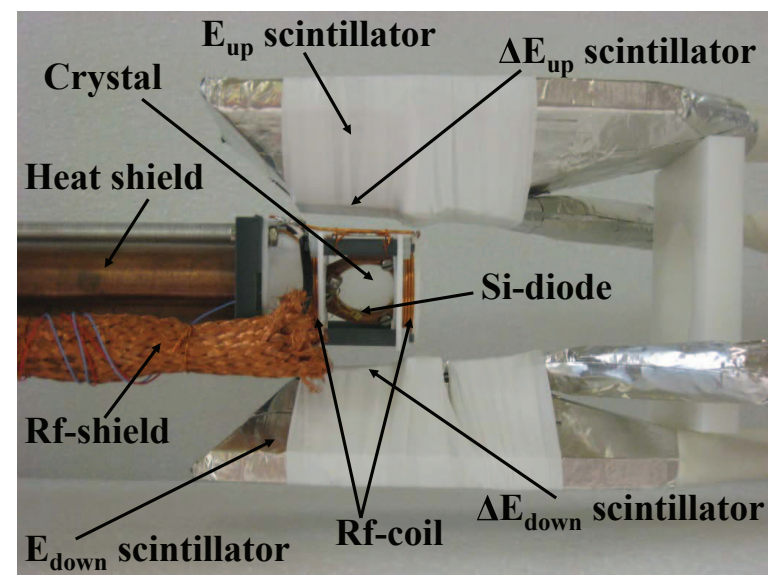

Fig. 3. Upstream view of the cold finger crystal holder.

On the cold finger crystal holder, a crystal with a diameter of $2 \mathrm{~cm}$ is mounted in a $\mathrm{Cu}$ holder, the thermal contact between both is made with thermal grease (Apiezon-N). A Cu holder is used since this material has a very high thermal conductivity: $401 \mathrm{~W} / \mathrm{mK}$ for $273.2 \mathrm{~K} \mathrm{[18]} \mathrm{and} \mathrm{even} \mathrm{more} \mathrm{for} \mathrm{lower} \mathrm{tempera-}$ tures. The crystal holder is then attached to the cold finger, a $\mathrm{Cu}$ bar which is directly cooled with liquid He using a continuous flow He-cryostat (Oxford Instruments, project $\mathrm{nr} 41580$ ). Temperatures as low as $12 \mathrm{~K}$ can be obtained at the end of the cold finger which is surrounded by a $\mathrm{Cu}$ heat shield.

The temperature on the crystal is much higher since it is placed in an unshel- 
tered room-temperature environment. The lowest temperature obtained for a $2 \mathrm{~mm}$ thick NaCl-crystal is $72.4 \mathrm{~K}$. The crystal temperature is measured with a Si diode sensor (LakeShore, DT-670C-SD, $0.1 \mathrm{~K}$ precision) which is mounted on the back side of the crystal and which can only be used in the absence of a rf-field.

\subsection{The rf-circuit}

The rf-circuit consists of a coil in which the rf-field is applied, one or more function generators, a sum box (in the case of $\beta$-NQR) and a rf-amplifier. A schematic overview of the rf-circuit with three generators is given in Fig. 4.

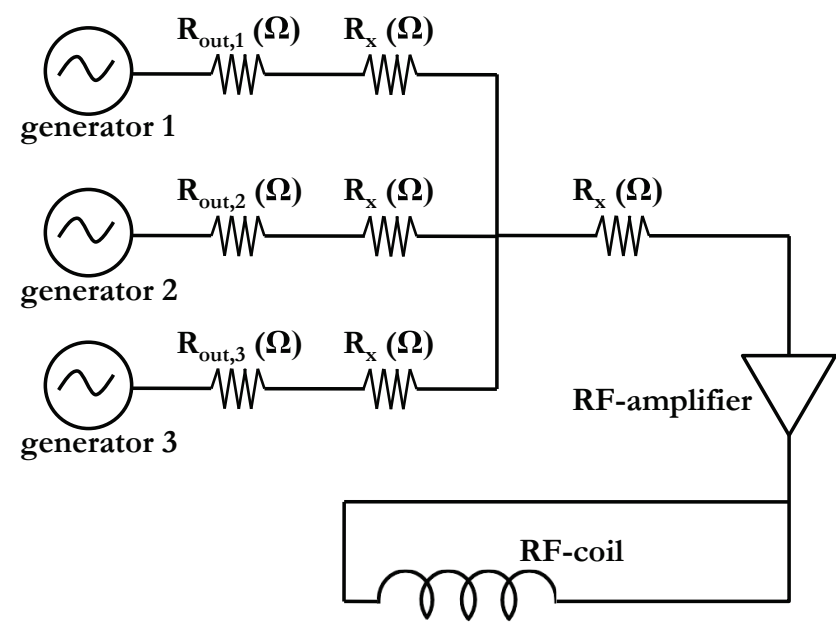

Fig. 4. Schematic overview of a $\beta$-NQR rf-circuit for three signals. The circuit can be extended for an arbitrary number of frequencies using more generators, expanding the electronics scheme and adjusting the values of $R_{x}$. For $\beta$-NMR, only one generator is used.

The rf-frequency scan is controlled by the Labview application which is used to set the applied frequencies with their respective amplitude, measuring time and modulation range. An rf-signal corresponding to these specifications is produced by an Agilent arbitrary waveform generator (model 33120A) which has an output impedance $\left(R_{\text {out }}\right)$ of $50 \Omega$. This type of generator cannot combine different frequencies and modulations in one signal. Therefore, several generators are used to perform a $\beta$-NQR measurement and all signals are combined into one output that is sent to a rf-amplifier with a $50 \Omega$ input impedance. The superposition of the different signals is done according to a simple electronics scheme, illustrated for three frequencies in Fig. 4. The value of the resistors $R_{x}$ is chosen as such to obtain a total resistance of $50 \Omega$, matching the input impedance of the rf-amplifier, over the combination of all generator output impedances $R_{\text {out }}$ and resistors $R_{x}$. Therefore, three signals require $R_{x}$ to be $25 \Omega$ while $R_{x}$ is $33.3 \Omega$ for a combination of five signals. 
The output signal of the function generator(s) is too weak to induce a sufficient rf-field strength in the coil. A first option to realize the necessary amplification is the use of a LRC-circuit, tuned at resonance in the proper frequency range by adjusting the variable capacity. This implies strong limitations on the accessible g-factor region as the available rf-frequency range only covers the width of the LRC-resonance. Another option to achieve the required rf-strength, is the introduction of an rf-amplifier. As the impedance of the coil (proportional to the rf-frequency) hardly ever matches the $50 \Omega$ output impedance of a standard amplification device, the rf-amplifier $800 \mathrm{~A} 3(800$ watts $\mathrm{CW}, 10 \mathrm{kHz}-3 \mathrm{MHz}$ ), manufactured by AR, is installed. This amplifier model is especially designed for applications with a variable output impedance, such as an rf-circuit with coil. For a fixed gain and by changing the amplitude of the function generator signal for each individual frequency, the current in the rf-circuit and consequently the rf-field strength inside the coil can be kept constant. This avoids the use of a resonant LRC-circuit and allows very broad rf-frequency scans to be performed. An AC-current of $500 \mathrm{~mA}$ peak to peak (pp) in the rf-circuit is sufficient for a $\beta$-NMR/ $\beta$-NQR measurement as it generates a rf-field with an amplitude between $0.1 \mathrm{mTpp}$ and $1 \mathrm{mTpp}$, depending on the specifications of the coil. During the run, the current in the rf-circuit is continuously monitored by an AC current probe (Tektronix, model P6021).

The output of the amplifier is connected to the rf-coil inside the vacuum chamber via a coaxial vacuum feedthrough. Along the complete trajectory, the coaxial cable is shielded with a $1.5 \mathrm{~mm}$ thick $\mathrm{Cu}$-layer. Since the penetration depth of eddy currents caused by rf-fields with a frequency $\nu$ is given by $\delta=1 / \sqrt{\pi \sigma \mu \nu}$ [19], $\mathrm{Cu}$ is chosen as a shielding material as it has a very high electric conductivity $\left(\sigma_{\mathrm{Cu}}=59.77 \times 10^{6} \mathrm{~S} / \mathrm{m}[20]\right) .99 \%$ of electromagnetic waves with a frequency of $100 \mathrm{kHz}$ is stopped by a Cu-layer of $1.03 \mathrm{~mm}$ while even less material is required for higher frequencies. Measurements with a pick-up coil show that the $\mathrm{Cu}$-shield around the coaxial cable reduces the rf-background by a factor of 5 to 8 . Note that a leakage of magnetic flux spreading outwards from the coil is still present, as the coil itself cannot be shielded. However, the effect of such a rf-stray field has a negligible impact on the efficiency of the system.

In the $\beta$-NMR/ $\beta$-NQR experiments, coils with a self-inductance of $L=22.1 \mu \mathrm{H}$ (Fig. 2) and $L=1.6 \mu \mathrm{H}$ (Fig. 3) are used. They respectively have $2 \times 20$ turns of $\mathrm{Cu}$-wire with a diameter of $0.7 \mathrm{~mm}$ and $2 \times 4$ turns with a diameter of $0.8 \mathrm{~mm}$. Between both halves of the coil, a space of about $2 \mathrm{~cm}$ is foreseen for the beam to be implanted in the crystal.

The strong $10 \mathrm{kHz}$ to $3 \mathrm{MHz}$ rf-signal generated in the rf-circuit in combination with the magnetic field up to $1.2 \mathrm{~T}$ allows to study a wide range of g-factors $(0.02<\mathrm{g}<4.0)$ and spectroscopic quadrupole moments. Both, the magnet and the rf-amplifier, make the $\beta$-NMR/ $\beta$-NQR setup accurate and powerful. 


\subsection{The scintillation detectors}

The $\beta$-particles are detected by two pairs of scintillation detectors along the magnetic field direction, one is situated above the crystal, the other below. Each pair consists of a thin $\Delta E$ detector, which is a rectangular prism with dimensions $30 \times 30 \times 2 \mathrm{~mm}^{3}$ and a $28 \mathrm{~mm}$ thick polyhedral E detector with bases of $80 \times 70 \mathrm{~mm}^{2}$ and $30 \times 55 \mathrm{~mm}^{2}$. A total geometric efficiency of $19 \%$ is obtained for high energetic $\beta$-particles emitted from the center and detected in coincidence between the two upper or the two lower detectors. All detectors are polystyrene scintillators (organic scintillation material PS89) and are covered with white Teflon tape and aluminium foil to optimize internal reflection and to avoid light leakage. The detection setup is included in Fig. 3.

To avoid scattering and losses of the $\beta$-particles, the scintillators are placed close to the crystal inside the vacuum chamber. For each $E$-detector, the scintillation light is transported through a straight plexiglass light guide $(44.2 \mathrm{~cm}$ long) to a photomultiplier tube (PMT) which is situated outside the vacuum chamber in an atmospheric environment. For the $\Delta E$-detectors, the light guides are bent, allowing a more compact geometry. The curved part of each light guide is constructed with 59 optic fibers ( $1 \mathrm{~mm}$ diameter) and is attached to a straight plexiglass piece of $35.5 \mathrm{~cm}$ long which is used to exit from the vacuum and to make the connection with the PMT. Four $20 \times 20 \mathrm{~cm}^{2}$ PMT's, type R7600U manufactured by Hamamatsu, are used as readouts. As can be deduced from the length of the light guides, they are placed at a distance of about $50 \mathrm{~cm}$ from the center in order to minimize the influence of the magnetic field $B_{0}$. At this distance, a magnetic field of less than $0.01 \mathrm{~T}$ remains if $1.2 \mathrm{~T}$ is applied in the center. Nevertheless, all photomultipliers are surrounded by a $\mu$-metal foil, to protect them from stray fields.

To determine the $\beta$-asymmetry in a $\beta$-NMR/ $\beta$-NQR measurement, coincidences between the upper two or the lower two detectors are required. This condition strongly reduces scattering and noise events in the $\beta$-spectra.

Outside the vacuum chamber, two germanium detectors are placed to register $\gamma$-rays. Each detector is positioned in front of a window where the thickness of the aluminium vacuum chamber is only $3 \mathrm{~mm}$ instead of the normal $2 \mathrm{~cm}$. The recorded $\gamma$-spectra contain lines following the $\beta$-decay of the isotopes implanted in the crystal and are therefore very useful for the beam identification. Also $\gamma$-decaying isomeric states present in the isotopes of interest can be observed provided that these levels are populated in the projectile-fragmentation reaction. 


\section{GEANT4 application}

\subsection{Description}

For the new $\beta$-NMR/ $\beta$-NQR setup, a GEANT4 Monte Carlo simulation code was developed (using version GEANT4.8.1 [21]). This GEANT4 application allows us to determine the $\beta$-detection efficiency of the setup and to describe the sensitivity of the setup for the detection of a resonant change in the $\beta$ asymmetry. Simulations in various experimental conditions were made, examining the influence of magnetic fields and $Q_{\beta}$-values. Also the impact of extra material in the vacuum chamber and the influence of the thickness and the tilting angle of the implantation crystal are determined.

In the GEANT4 application, two full-scale geometric configurations have been instantiated, one for each crystal holder (see section 3). Both detection geometries are constructed with predefined GEANT4 building blocks inside a 'world volume' of $220 \times 230 \times 114 \mathrm{~mm}^{3}$ (see Fig. 5). The four $\beta$-detectors are defined as 'Sensitive Detectors' which keep track of the energies deposited in the scintillation material and the number of interacting particles during the run. A uniform magnetic field can be applied in the Z-direction. The rf-field inside the coil is small (maximum $1 \mathrm{mTpp}$ ) and therefore neglected in the simulation.
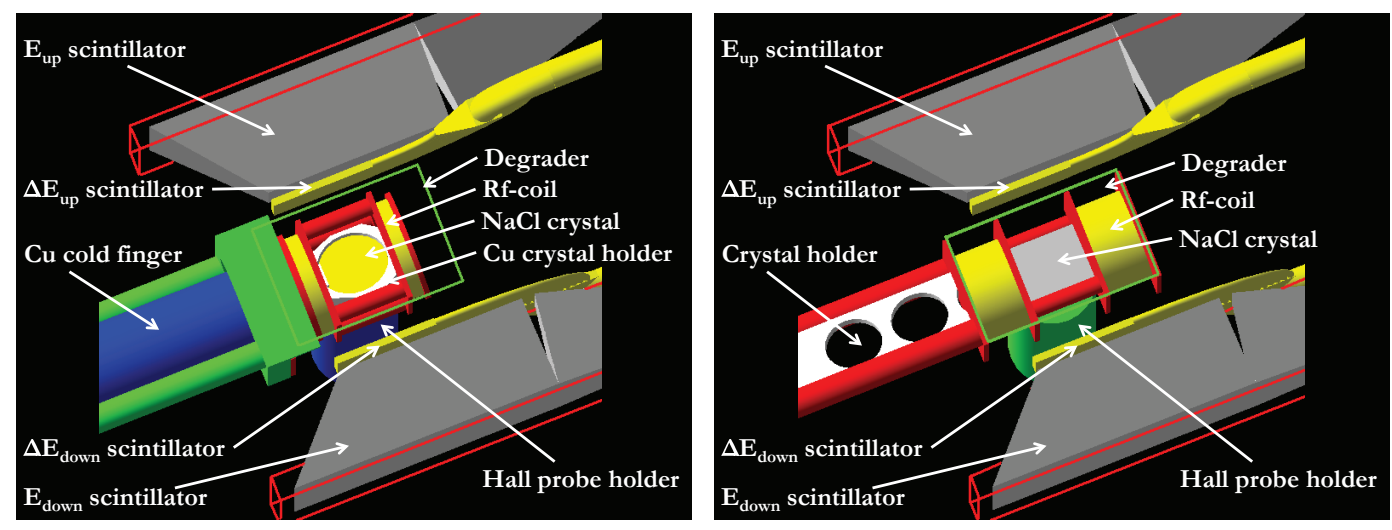

Fig. 5. GEANT4 geometries for the cold finger (left) and the room temperature (right) crystal holder.

In the center of the implemented GEANT4 geometries, a point-like source is placed which simulates the $\beta$-decay of the implanted nuclei. The probability to have allowed $\beta^{-}$-decay at a certain energy and for a particular nucleus, given by its mass A and proton number Z, is described by D.H. Wilkinson in Refs. [22-26]. The methodology outlined in that work is used here to define the $\beta$-spectrum generated by the source. The simulated $\beta$-spectrum has one particular $Q_{\beta}$-value and does not take into account the specific populations of the states in the daughter nucleus. Electrons are emitted under an angle $\theta$ 
with a probability determined by the angular distribution function $\mathrm{W}(\theta)$ (see Eq. (1)). The implantation of the polarized beam in the crystal is not included in the simulation.

The interactions and trajectories of all primary and secondary particles are simulated using the standard low energy GEANT4 libraries [27] which include ionization, bremsstrahlung, multiple scattering, rayleigh and compton scattering, photo electric effects and $\gamma$-conversion for energies between $150 \mathrm{eV}$ and $100 \mathrm{GeV}$.

In each simulation, two runs are executed, one with an anisotropic $\beta$-decay pattern, the other with an isotropic distribution of the $\beta$-radiation. In both runs, 100 million events are processed. Each event starts with a primary particle, released by the source with a particular energy and in a certain direction according to the conditions described above. A Monte Carlo simulation is made of the transport and the interactions of this particle and the created secondary particles with the material and the magnetic field in the "world volume'. When the event interacts with one of the scintillation detectors, the deposited energy is registered. After each run, the detected $\beta$-spectra are reconstructed as shown in Fig. 6.
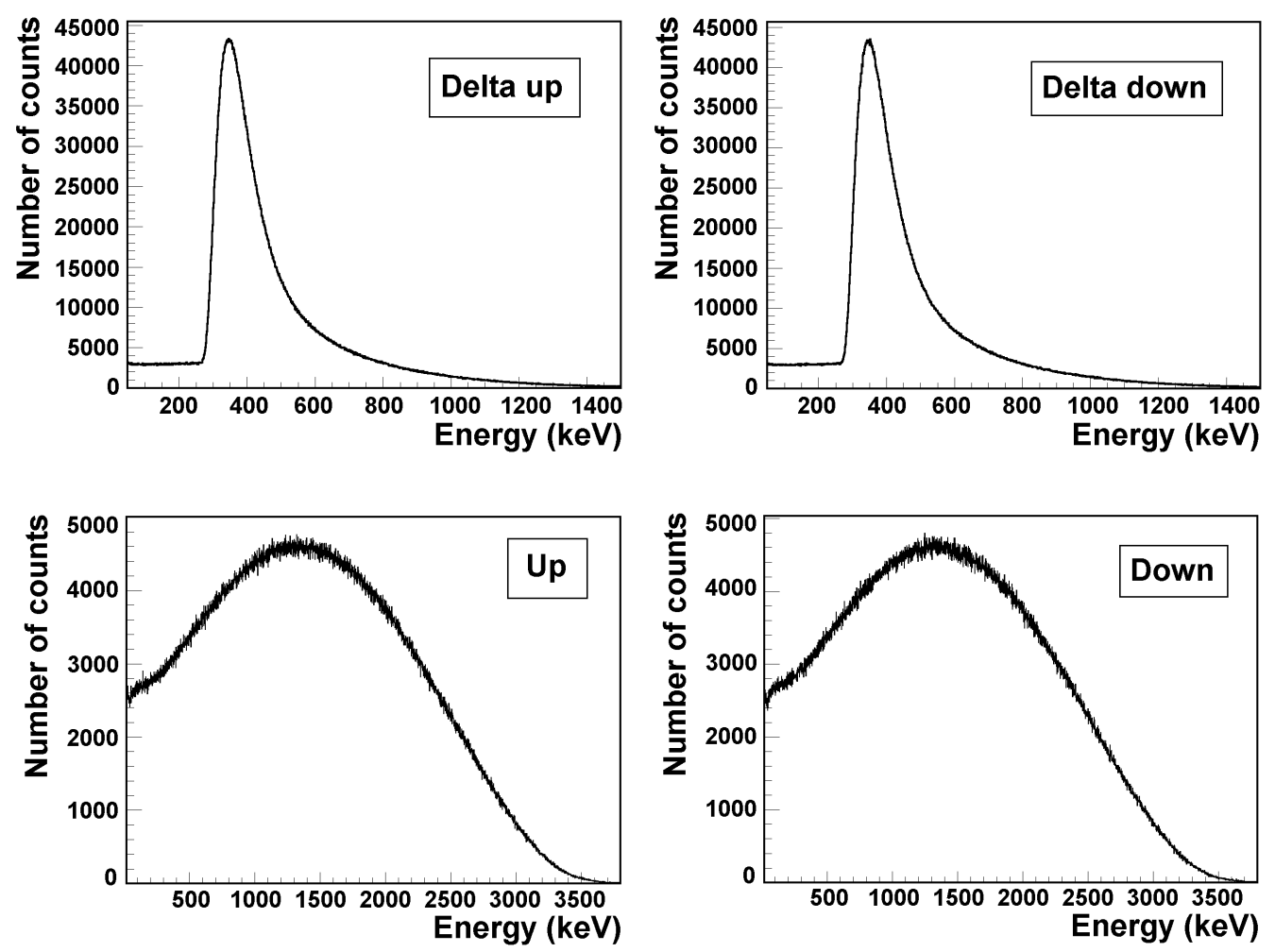

Fig. 6. Simulated $\beta$-spectra for a GEANT4 geometry including only the detectors and using a ${ }^{41} \mathrm{Cl} \beta^{-}$-source $\left(Q_{\beta}=3860 \mathrm{keV}\right)$ in the center of the vacuum chamber.

The number of detected particles in the different scintillators and coincidences is used to calculate the detection efficiency, the $\beta$-asymmetry, the observed $\beta$ - 
NMR/ $\beta$-NQR effect (i.e. the $\beta$-asymmetry change) and the observed fraction of the full $\beta$-NMR/ $\beta$-NQR amplitude. The detection efficiency $\epsilon_{i}$ of a particular detector (combination) $i$ is defined as the number of detected events $N_{i}$ divided by the total number of processed events $N$ (100 million). It is obvious that only data from the run with an isotropic radiation pattern can be used to calculate $\epsilon_{i}$.

$$
\epsilon_{i}=\frac{N_{i}}{N}
$$

The $\beta$-asymmetry (Eq. 2) can be calculated for an anisotropic and an isotropic radiation pattern. According to Eq. $2, A_{\text {aniso }} \simeq\left|A_{\beta} P\right|$ while theoretically, $A_{\text {iso }}$ should be zero. To obtain the observed $\beta$-asymmetry change $\Delta$ (proportional to the observed $\beta$-NMR/ $\beta$-NQR amplitude), the simulated anisotropic and isotropic $\beta$-asymmetries are subtracted.

$$
\Delta=\left|A_{\text {aniso }}-A_{\text {iso }}\right|
$$

A comparison of the simulated $\beta$-NMR/ $\beta$-NQR amplitude $\Delta$ with its theoretical value $\left|A_{\beta} P\right|$ gives the sensitivity $\delta$ of the setup to observe a resonant change in the $\beta$-asymmetry. A perfect setup leads to $\delta=1$ (=100\%).

$$
\delta=\frac{\Delta}{\left|A_{\beta} P\right|}
$$

The values of $\epsilon_{i}, \Delta$ and $\delta$ depend on several parameters: how much scattering and absorption are induced by the material present in the vacuum chamber, the density, the tilting and the thickness of the implantation crystal, the $Q_{\beta}$ of the decaying nuclei and the strength of the applied magnetic field $B_{0}$. The influence of these parameters on $\epsilon_{i}$ and $\delta$ is studied in the following four subsections.

All Geant4 simulations quoted in this work are performed for ${ }^{41} \mathrm{Cl}(Z=17)$. The ground-state spin is assumed to be $I=1 / 2$ and $Q_{\beta}$ is taken as $3860 \mathrm{keV}$. The real $Q_{\beta}$ of ${ }^{41} \mathrm{Cl}$ is $5728(65) \mathrm{keV}$ but more than $80 \%$ of the $\beta$-decay goes to the $1868 \mathrm{keV}$ level $\left(I=1 / 2^{+}\right)$in ${ }^{41} \mathrm{Ar}$ [28], making $Q_{\beta}=3860 \mathrm{keV}$ a more realistic value. The estimated $A_{\beta}$-parameter for a pure Gamow-Teller $1 / 2^{+} \rightarrow$ $1 / 2^{+}$transition is $2 / 3$ [14]. An arbitrary polarization $P$ of $10 \%$ is assumed.

\subsection{Scattering and absorption due to extra elements in the vacuum chamber}

When only the four $\beta$-detectors are present in the geometry, the detection efficiency of the $E$-detectors ( $10.3 \%$ per detector) is higher than that of the $\Delta E$-scintillators $(9.7 \%$ per detector). This is due to the significantly larger detection area of the $E$-detectors compared to the small $\Delta E$-surface. As indicated in Fig. 1, $\beta$-particles emitted from the center of the geometry can 
reach the $E$-detector without passing the $\Delta E$-scintillator first. The efficiency to detect a coincident event between the $E$ and $\Delta E$-detectors $(7.3 \%$ per detector combination) is even more reduced since the lowest-energy $\beta$-particles are stopped in the $\Delta E$ scintillation material.

Table 2 shows the simulated values for the detection efficiency of the up and the down coincidence spectra, the $\beta$-asymmetry for an isotropic ensemble and the observed fraction of the full $\beta$-NMR/ $\beta$-NQR amplitude for various geometric configurations. All simulations were made with the cold finger GEANT4 application and $A_{\text {iso }}$ as well as $\delta$ were calculated using only coincident events.

Table 2

Simulations performed for various geometric configurations of the cold finger setup, resulting in the efficiency for coincidence detection in the upper $\left(\epsilon_{\text {coinc-up }}\right)$ and lower $\left(\epsilon_{\text {coinc-down }}\right)$ detector pair, the observed asymmetry for an isotropic $\beta$-pattern $\left(A_{\text {iso }}\right)$ and the sensitivity to observe a resonant change in the $\beta$-asymmetry $(\delta)$.

\begin{tabular}{|l|c|c|c|c|}
\hline Elements included in geometry & $\epsilon_{\text {coinc-up }}$ & $\epsilon_{\text {coinc-do }}$ & $A_{\text {iso }}(\%)$ & $\delta(\%)$ \\
\hline \hline detectors only & $7.32 \%$ & $7.32 \%$ & -0.0074 & 91.08 \\
\hline detectors + lightguides & $7.29 \%$ & $7.29 \%$ & -0.035 & 90.84 \\
\hline " + degrader + coil + hall probe & $7.68 \%$ & $7.67 \%$ & 0.055 & 88.08 \\
\hline " + crystal holder @ 0 & $4.68 \%$ & $4.68 \%$ & -0.0079 & 83.64 \\
\hline " $+\mathrm{NaCl}, 1 \mathrm{~mm} @ 0^{\circ}$ & $3.24 \%$ & $3.24 \%$ & 0.0059 & 67.94 \\
\hline
\end{tabular}

As can be seen in Table 2, the detection efficiency changes significantly when the crystal holder and the crystal are brought into the geometry. Other parts (e.g. the hall probe, the lightguides, the rf-coil, the degrader, ... ) do not seem to have a large influence on the scattering and absorption of the $\beta$-particles and therefore on $\epsilon$. When only the scintillators are included in the setup, the total coincidence detection efficiency $(14.6 \%)$ is smaller than the full geometric efficiency (19\%). This is due to the low $Q_{\beta}$-value used in the calculations.

For the simulations presented in Table $2, A_{i s o}$ has values close to zero, as expected for a symmetric setup. As soon as a tilted implantation crystal or another geometric asymmetry (e.g. the $\beta$-source not exactly in the center) is brought into the system, $A_{\text {iso }}$ starts to deviate. This induces an anisotropic radiation pattern even when no polarization is present. $A_{i s o}=0.30 \%$ and $A_{\text {iso }}=0.59 \%$ have been obtained for a $1 \mathrm{~mm}$ thick $\mathrm{NaCl}$ crystal tilted by $-15^{\circ}$ and $-30^{\circ}$ respectively with respect to the vertical axis.

The extended solid angle of the $\beta$-detectors makes that only $91 \%$ of the full $\beta$-asymmetry change is observed for a geometry which exclusively consists of the four $\beta$-scintillators. For the complete $\beta$-NMR/ $\beta$-NQR configuration with a NaCl-crystal of $1 \mathrm{~mm}$ thickness, $\delta$ is reduced to about $70 \%$. This is mostly due to scattering and absorption of $\beta$-particles.

The simulation results obtained with the cold finger and the normal crys- 
tal holder geometry are equivalent and do not show any specific differences. Although the cold finger geometry contains more material, the detection efficiency is at most $0.3 \%$ lower than the one determined with the room temperature crystal holder. The fraction of the full $\beta$-asymmetry change observed with the cold finger setup is at most $1.5 \%$ less than $\delta$ determined with the room temperature crystal holder geometry in exactly the same conditions.
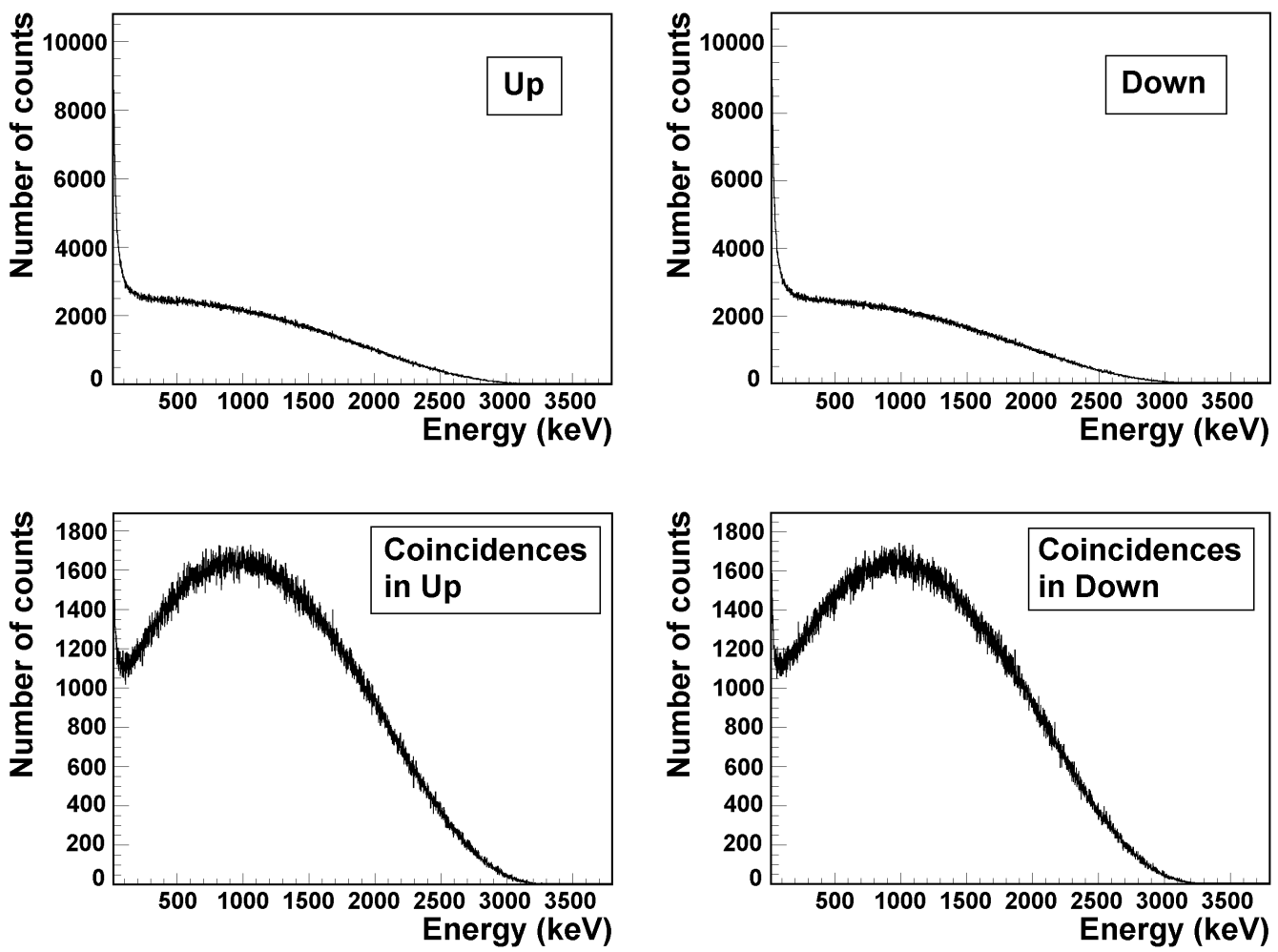

Fig. 7. Simulated $\beta$-spectra of the E-up/E-down detectors, with and without the coincidence condition. The simulations were performed with the cold finger geometry using a $1 \mathrm{~mm}$ thick $\mathrm{NaCl}$ crystal $\left(\right.$ at $\left.0^{\circ}\right)$.

Including all vacuum-chamber elements in the GEANT4 geometry has an effect on the unconditioned $\beta$-spectra of both $E$-scintillators (see Fig. 7 ). They clearly show a low-energy component $(<150 \mathrm{keV})$ in the simulated signals of the $E$-scintillators (to be compared to Fig 6 where no such tail is present). Since the $\beta$-particles suffer from scattering and energy absorption in the implantation crystal and crystal holder, a significant fraction reaches the detectors at low energy. Most of them are stopped in the $\Delta E$-material. However, as the $\Delta E$-detector does not fully cover the $E$-surface, low-energy $\beta$-particles are also recorded in the $E$-detector, inducing a strong low-energy component in the unconditioned $\beta$-spectra. By requiring a coincidence with the respective $\Delta E$-detectors, as shown in Fig. 7 , the low-energy component is significantly reduced. 


\subsection{Properties of the implantation crystal}

The impact of the crystal thickness and the tilting angle on $\epsilon$ and $\delta$ is shown in Fig. 8. Due to the absorption in the crystalline medium, an increase of the crystal thickness from $1 \mathrm{~mm}$ to $2 \mathrm{~mm}$ significantly reduces the detection efficiency and the observed asymmetry change. In both cases, tilting the implantation material yields better results since it minimizes the effective path lenoth of the narticles towards the B-detectors
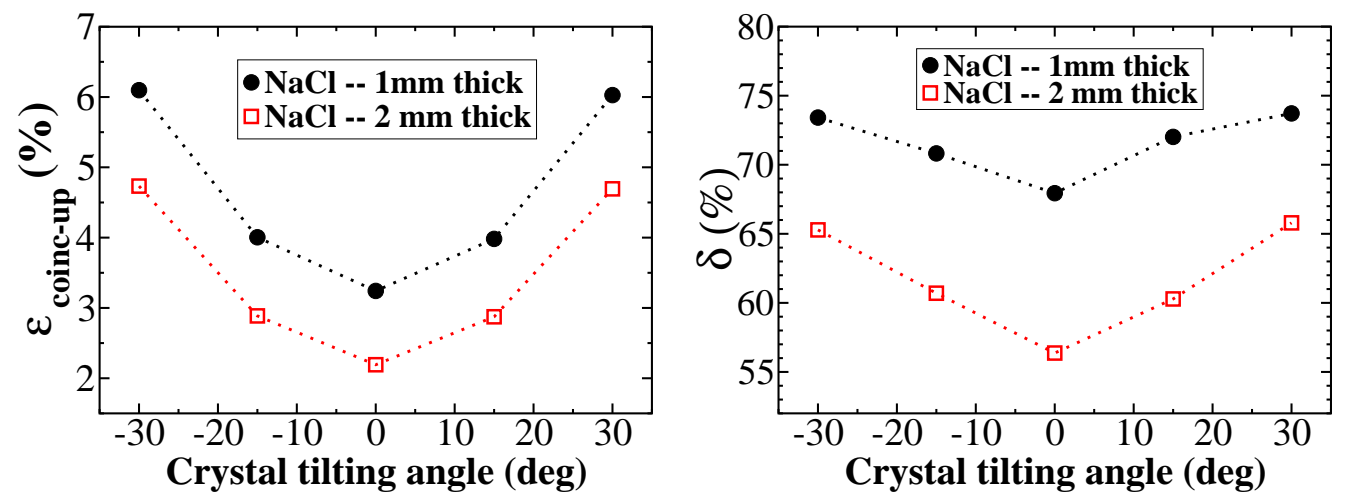

Fig. 8. Efficiency of coincident detection in the upper detector pair $\left(\epsilon_{\text {coinc-up }}\right)$ and sensitivity to observe a resonant change in the $\beta$-asymmetry $(\delta)$ for two different crystal thicknesses $\left(1\right.$ and $2 \mathrm{~mm}$ ). The simulations were made for ${ }^{41} \mathrm{Cl}$ with $Q_{\beta}=3860 \mathrm{keV}, P=10 \%$ and the complete cold finger setup.

All simulations shown so far are made with a NaCl-crystal. Changing the material to e.g. Si can have an influence on the detection efficiency and the observed $\beta$-asymmetry change. Table 3 shows a comparison between a $\mathrm{NaCl}$ and a $\mathrm{Si}$ crystal of $1 \mathrm{~mm}$ and tilted over $-30^{\circ}$ with respect to the vertical axis. As the density of $\mathrm{Si}\left(2.328 \mathrm{~g} / \mathrm{cm}^{3}\right.$ [20]) is slightly higher than the density of $\mathrm{NaCl}\left(2.168 \mathrm{~g} / \mathrm{cm}^{3}[29]\right)$, its increased stopping power results in a reduced $\epsilon$ and $\delta$.

Table 3

Simulations performed for a $\mathrm{NaCl}$ and a Si-crystal in the cold finger geometry, resulting in the efficiency for coincidence detection in the upper $\left(\epsilon_{\text {coinc-up }}\right)$ and lower $\left(\epsilon_{\text {coinc-down }}\right)$ detector pair, the observed asymmetry for an isotropic $\beta$-pattern $\left(A_{\text {iso }}\right)$ and the sensitivity to observe a resonant change in the $\beta$-asymmetry $(\delta)$. The $\beta$-spectrum of ${ }^{41} \mathrm{Cl}$ was used with $Q_{\beta}=3860 \mathrm{keV}$ and $P=10 \%$.

\begin{tabular}{|l|c|c|c|c|}
\hline Configuration & $\epsilon_{\text {coinc-up }}$ & $\epsilon_{\text {coinc-do }}$ & $A_{\text {iso }}(\%)$ & $\delta(\%)$ \\
\hline \hline $\mathrm{NaCl}, 1 \mathrm{~mm} @-30^{\circ}$ & $6.10 \%$ & $6.03 \%$ & 0.59 & 73.42 \\
\hline $\mathrm{Si}, 1 \mathrm{~mm} @-30^{\circ}$ & $5.16 \%$ & $5.11 \%$ & 0.44 & 67.25 \\
\hline
\end{tabular}



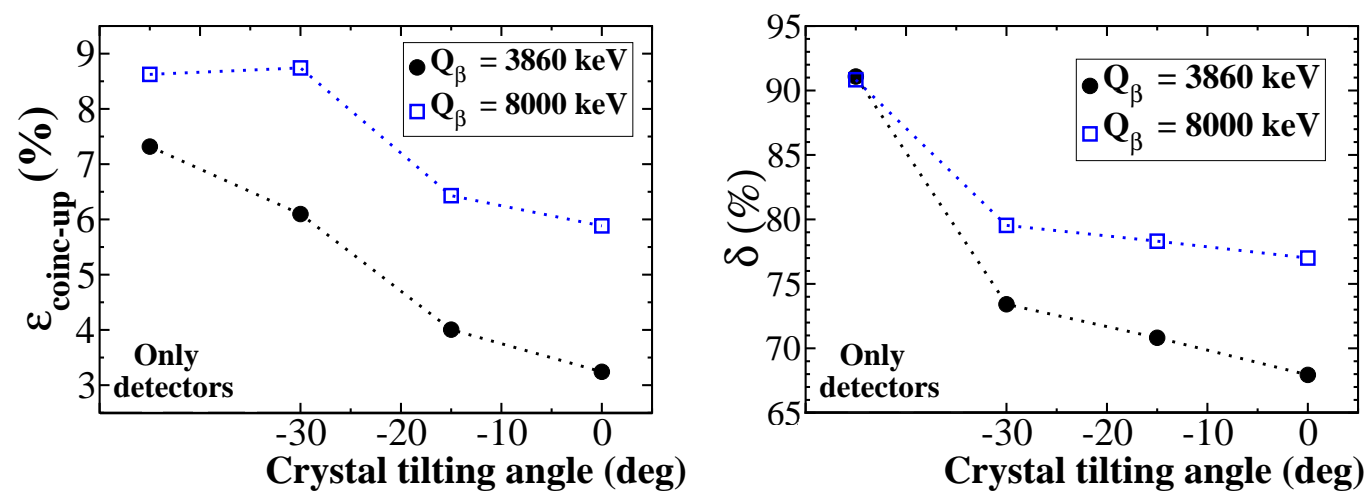

Fig. 9. Efficiency of coincident detection in the upper detector pair $\left(\epsilon_{\text {coinc-up }}\right)$ and sensitivity to observe a resonant change in the $\beta$-asymmetry $(\delta)$ for two different $Q_{\beta}$-values $(3860 \mathrm{keV}$ and $8000 \mathrm{keV})$. The simulations were made for ${ }^{41} \mathrm{Cl}, P=10 \%$ and the complete cold finger setup, including a $1 \mathrm{~mm}$ thick NaCl-crystal.

Fig. 9 shows a comparison between simulations performed for $\epsilon$ and $\delta$ applying two values of the $\beta$-endpoint energy, $Q_{\beta}=3860 \mathrm{keV}$ and $Q_{\beta}=8000 \mathrm{keV}$. The cold finger geometry was used with a NaCl-crystal of $1 \mathrm{~mm}$. A higher endpoint energy results in an increased amount of $\beta$-particles passing through the $\Delta E$-detectors. Therefore it leads to a more efficient $\beta$-detection in the $E$-scintillators and a higher coincidence rate.

When only the detectors are included in the GEANT4 geometry, the efficiency $\delta$ to observe the $\beta$-asymmetry change is similar for both $Q_{\beta}$-values (Fig. 9). As soon as the complete setup is taken into account, $\delta$ increases with increasing endpoint energy. High-energy $\beta$-particles do not easily loose their polarization in scattering processes.

For all experimental conditions, one can conclude that the detection efficiency and the observed asymmetry change improve when the $\beta$-decay has a higher $Q_{\beta}$-value.

High $Q_{\beta}$-energies also drastically change the unconditioned and the coincident $\beta$-spectra as illustrated in Fig. 10. Simulations performed for $Q_{\beta}=12 \mathrm{MeV}$ show a clear $\Delta E$ peak around $5.5 \mathrm{MeV}$. This peak contains the signals of high energetic electrons which pass through both detectors.

\subsection{The impact of magnetic fields}

When a high static magnetic field is applied in the Z-direction, charged particles start spiraling around the magnetic field lines in the direction of the $\beta$ detectors. This process results in a significantly higher detection efficiency but a much lower sensitivity to the $\beta$-asymmetry change. Simulations with $B_{0}=1 \mathrm{~T}$ and without magnetic field have been performed for ${ }^{41} \mathrm{Cl}\left(Q_{\beta}=3860 \mathrm{keV}\right.$, 

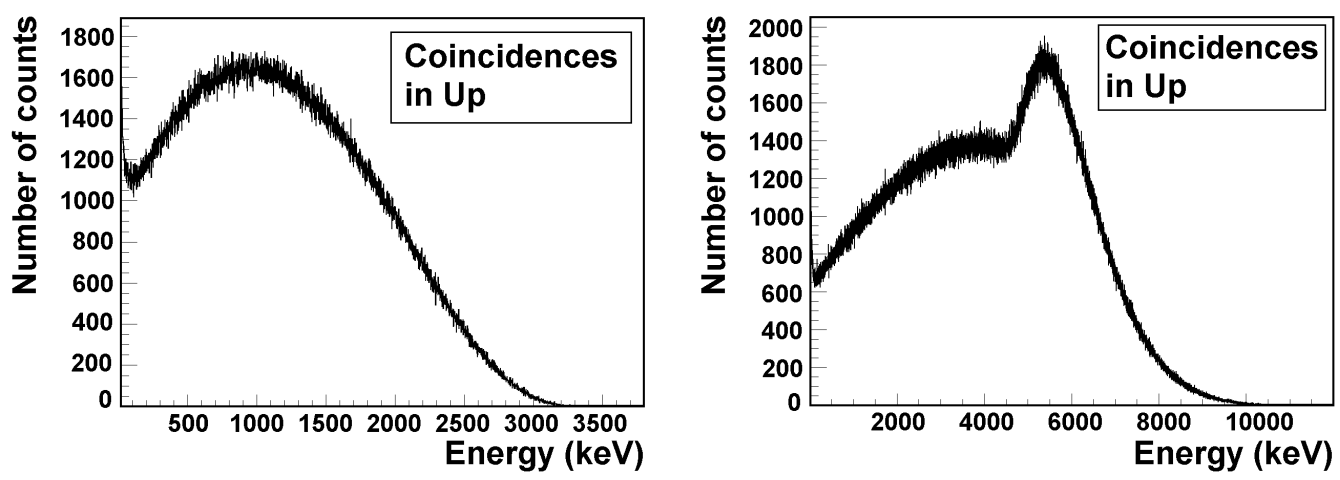

Fig. 10. Coincident $\beta$-spectra of the E-up detector conditioned with $\Delta E$-up for $Q_{\beta}=3860 \mathrm{keV}$ and $Q_{\beta}=12 \mathrm{MeV}$. The simulations were performed for the full cold finger setup with a $1 \mathrm{~mm}$ thick $\mathrm{NaCl}$ crystal.

$P=10 \%)$ and the results are given in Fig. 11. Coincidence detection efficiencies as high as $14 \%$ in one detector set have been observed in a magnetic field of $1 \mathrm{~T}$ (NaCl-crystal of $1 \mathrm{~mm}$ and tilted over $\left.-30^{\circ}\right)$ but they can only barely compensate for the $50 \%$ loss in the sensitivity to observe the total $\beta$-NMR/ $\beta$ NQR amplitude.
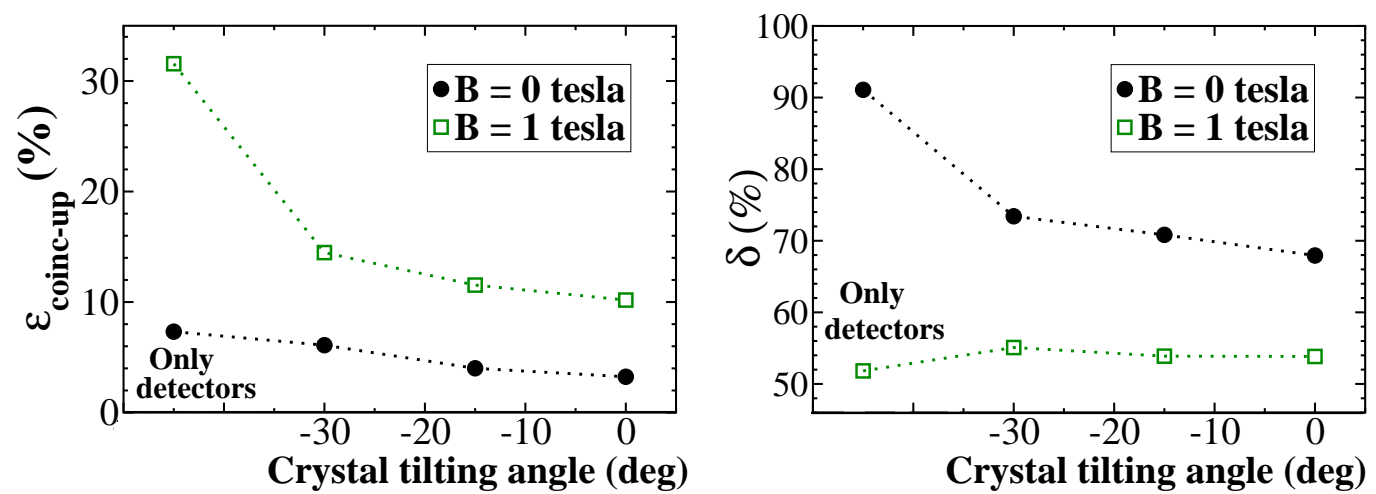

Fig. 11. Efficiency of coincident detection in the upper detector pair $\left(\epsilon_{\text {coinc-up }}\right)$ and sensitivity to observe a resonant change in the $\beta$-asymmetry $(\delta)$ without magnetic field and for $B_{0}=1 \mathrm{~T}$. The simulations were made for ${ }^{41} \mathrm{Cl}\left(Q_{\beta}=3860 \mathrm{keV}, P=10 \%\right)$ in a $\mathrm{NaCl}$-crystal of $1 \mathrm{~mm}$.

\section{Conclusions}

The detailed description of a new $\beta$-NMR/ $\beta$-NQR setup developed to be installed at the final focal point of the LISE fragment separator at GANIL demonstrates that it is now possible to measure a broad range of g-factors $(0.02<\mathrm{g}<4.0)$ and spectroscopic quadrupole moments. Two main components 
are responsible for this: the strong and homogeneous magnet and the rfamplifier especially designed for variable output impedance circuits.

A theoretical description of the setup has been made using the GEANT4 toolkit. Monte Carlo simulations have been carried out, probing the influence of various elements on the detection efficiency and on the expected fraction of the total $\beta$-NMR/ $\beta$-NQR amplitude. The effects of the crystal thickness and tilting angle, the material in the vacuum chamber, magnetic fields and high $Q_{\beta}$-values have been extensively studied and a much better understanding of the experimental conditions has been obtained.

From the GEANT4 simulations, the ideal conditions to perform a $\beta$-NMR $/ \beta$ NQR experiment can be deduced. Mother nuclei with a high $Q_{\beta}$-value induce a large detection efficiency and a good sensitivity to the $\beta$-asymmetry change. The implantation crystal must be thin and tilted as much as possible with respect to the vertical axis. Applying a magnetic field $B_{0}$ induces a high detection efficiency but it reduces the sensitivity to observe the full $\beta$-NMR/ $\beta$ NQR amplitude. As both effects cancel each other, no strong influence of $B_{0}$ on the $\beta$-NMR/ $\beta$-NQR measurement is observed.

So far, three experiments have been performed with the new $\beta$-NMR/ $\beta$-NQR setup. The first experiment, in which the g-factors of ${ }^{17} \mathrm{~N}$ and ${ }^{18} \mathrm{~N}$ were remeasured, was a test run to evaluate the performance of the setup. Afterwards, several improvements were made which are included in the simulations made in this work. In the second and the third experiment, the g-factor of ${ }^{44} \mathrm{Cl}$ and the quadrupole moment of ${ }^{31} \mathrm{Al}$ were determined. The outcome of all experiments will be published elsewhere [30-32].

\section{Acknowledgements}

We are grateful to the GANIL staff for their technical support. This work has been financed by the European Community FP6 - Structuring the ERA - Integrated Infrastructure Initiative contract EURONS No. RII3-CT-2004506065, by the FWO-Vlaanderen and by the IAP-programme of the Belgium Science Policy under grand number P6/23.

\section{References}

[1] O. Sorlin and M.-G. Porquet, Prog. Part. Nucl. Phys. 61, 602 (2008)

[2] A. Kastler, J. Phys. Radium 11, 255 (1950)

[3] K. Asahi et. al., Phys. Lett. B 251, 488 (1990)

[4] H. Okuno et. al., Phys. Lett. B 335, 29 (1994) 
[5] D. Borremans et. al., Phys. Rev. C 66, 054601 (2002)

[6] D.E. Groh et. al., Phys. Rev. Lett. 90, 202502 (2003)

[7] K. Turzo et. al., Phys. Rev. C 73, 044313 (2006)

[8] A. Abragam, The Principles of Nuclear Magnetism, Clarendon, Oxford (1961)

[9] E. Matthias et. al. Phys. Rev. A 4, 1626 (1971)

[10] R. Anne et. al., Nucl. Instrum. Methods A 257, 215 (1987)

[11] R. Anne et. al., Nucl. Instrum. Methods B 70, 276 (1992)

[12] http://groups.nscl.msu.edu/lise/lise.html

[13] O.B. Tarasov and D. Bazin, Nucl. Instrum. Methods B 266, 4657 (2008)

[14] N.J. Stone and H. Postma (Eds.), Low-Temperature Nuclear Orientation (chapter 3), Elsevier, Amsterdam (1986)

[15] D. Borremans et. al., Phys. Rev. C 72, 044309 (2005)

[16] C.P. Slichter, Principles of Magnetic Resonance, Springer-Verlag, BerlinHeidelberg-New York (1978)

[17] T. Yamanishi et. al., J. Phys. Soc. Japan 64, 643 (1995)

[18] Y.S. Touloukian, R.W. Pavell, C.Y. Ho and P.G. Klemens, Thermophysical properties of matter, The TPRC Data Series Vol I, Thermal Conductivity Metallic Elements \& Alloys, IFI/PLENUM, New-York, Washington (1970)

[19] C.G. Someda, Electromagnetic Waves, Chapman \& Hall, London-WeinheimNew York-Tokyo-Melbourne-Madras (1998)

[20] R.E. Bolz and G.L. Tuve (Eds.), Handbook of tables for applied engineering science, the chemical rubber co., 18901 Cranwood Parkway, Cleveland Ohio 44128 (1970)

[21] GEANT collaboration, Nucl. Instrum. Methods A 506, 250 (2003)

[22] D.H. Wilkinson, Nucl. Instrum. Methods A 275, 378 (1989)

[23] D.H. Wilkinson, Nucl. Instrum. Methods A 290, 509 (1990)

[24] D.H. Wilkinson, Nucl. Instrum. Methods A 335, 305 (1993)

[25] D.H. Wilkinson, Nucl. Instrum. Methods A 365, 203 (1995)

[26] D.H. Wilkinson, Nucl. Instrum. Methods A 365, 497 (1995)

[27] http://www.ge.infn.it/geant4/lowE/index.html

[28] J.A. Cameron and B. Singh, Nuclear Data Sheets 94, 429 (2001)

[29] http://www.mateck.de/HiPuMa/e66e.asp.id.Sodium.html 
[30] M. De Rydt et. al., submitted to Phys. Rev. C (2009)

[31] M. De Rydt et. al., Phys. Lett. B 678, 344 (2009)

[32] M. De Rydt, to be submitted to Phys. Rev. C (2009) 